\title{
Efecto de los componentes del agua de mar durante el fenómeno de corrosión por picaduras del cobre ${ }^{(\bullet)}$
}

\author{
E. García O. ${ }^{(*)}, \mathrm{J}_{\text {. Uruchurtu }}{ }^{(*)}$ y J. Genescá ${ }^{(*)}$ \\ Resumen El cobre es el elemento principal de las aleaciones $\mathrm{Cu}-\mathrm{Ni}$ recomendadas para aplicaciones marinas. \\ En este trabajo se presenta el comportamiento electroquímico del cobre con los diferentes componen- \\ tes del agua de mar y el efecto de estos sobre la forma de ataque del cobre. Se observó que los iones \\ cloruro, en unión con los iones sulfato, son los principales causantes del fenómeno de corrosión por \\ picaduras, existiendo, además, una relación significativa entre el potencial crítico de picado $\left(E_{\mathrm{p}}\right)$ y la \\ relación $\left(\mathrm{Cl}^{-} / \mathrm{SO}_{4}^{2-}\right)$ de tipo parabólico, lo cual pone de manifiesto la importancia del ion sulfato en el \\ fenómeno de corrosión por picaduras como promotor de este tipo de mecanismo.
}

Palabras clave: Cobre. Agua de mar. Picaduras. Corrosión localizada.

\section{Effect of the sea water components in the pitting corrosion of copper}

\begin{abstract}
Copper is the main component in $\mathrm{Cu}-\mathrm{Ni}$ alloys recommended for sea water applications. This work presents the electrochemical behaviour of copper in presence of different sea water components and its influence on the corrosion attack. The combined action of chloride and sulphate ions is the main responsible factor for the localised corrosion attack in the form of pitting. A significant parabolic relation exists between the critical pitting potential $\left(E_{\mathrm{p}}\right)$ and the weight ratio of chloride to sulphate $\left(\mathrm{Cl}^{-} / \mathrm{SO}_{4}^{2-}\right)$ ions. Therefore sulphate ions is an important component for pitting corrosion of copper.
\end{abstract}

Keywords: Copper. Sea water. Pitting. Localised corrosion.

\section{INTRODUCCIÓN}

El cobre es el principal constituyente de las aleaciones $\mathrm{Cu}-\mathrm{Ni}$ de composición nominal típica 70-30 y 90-10 recomendadas para sistemas de enfriamiento que usan agua de mar como agente refrigerante. De aquí la importancia de la respuesta a la corrosión del cobre frente al agua de mar y a sus diversos constituyentes y, principalmente, al fenómeno de corrosión por picaduras que se puede presentar sobre el material.

La corrosión por picaduras en el cobre fue denominada por Cambell (1) como picadura nodular,

(•) Trabajo recibido el día 24 de marzo de 1995.

(*) Instituto de Investigaciones Eléctricas (IIE). Dpto. de Fisicoquímica Aplicada, Av. Reforma No. 133. Colonia Palmira. 62490 Temixco. Morelos (México).

(**) Facultad de Química. Universidad Nacional Autónoma de México (UNAM). Ciudad Universitaria. 04510 México D.F. debido a que los productos de corrosión se apilaban en sitios muy localizados formando nódulos o pequeños montículos (Fig. 1).

Cornwell et al. (2) propusieron el siguiente mecanismo de picaduras para el cobre en agua de mar, donde los iones $\mathrm{Cu}^{+} \mathrm{y} \mathrm{Cl}^{-}$reaccionan en sitios determinados siguiendo la reacción:

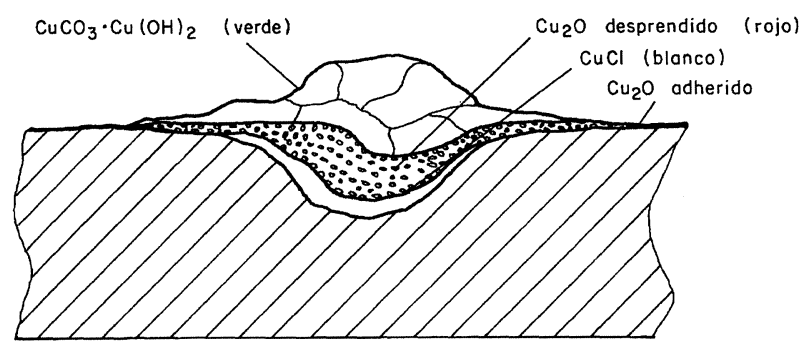

FIG. 1.- Sección transversal de una picadura de cobre (1).

Fig. 1.-Cross section of a copper pit (1). 


$$
\mathrm{Cu}^{+}+\mathrm{Cl}^{-} \Rightarrow \mathrm{CuCl}
$$

En dichos sitios o puntos de nucleación de la picadura, el $\mathrm{CuCl}$ sufre una hidrólisis formando $\mathrm{Cu}_{2} \mathrm{O}$, el cual precipita en la superficie del metal como lo expresa la siguiente reacción:

$$
2 \mathrm{CuCl}+\mathrm{H}_{2} \mathrm{O} \Rightarrow \mathrm{Cu}_{2} \mathrm{O}+2 \mathrm{HCl}
$$

La reacción catódica que soporta la disolución anódica es la reducción de oxígeno:

$$
\mathrm{O}_{2}+2 \mathrm{H}_{2} \mathrm{O}+4 e^{-} \Rightarrow 4 \mathrm{OH}^{-}
$$

Por dicha alcalinización, en las zonas catódicas y en presencia de bicarbonatos, se provoca la siguiente reacción:

$$
\mathrm{OH}^{-}+\mathrm{HCO}_{3}^{-} \Rightarrow \mathrm{CO}_{3}^{2-}+\mathrm{H}_{2} \mathrm{O}
$$

que causa la precipitación de carbonato de calcio y carbonatos básicos de cobre.

La corrosión por picaduras del cobre en medios acuosos se debe a la presencia de aniones agresivos, como son los cloruros, bromuros, tiosulfatos y sulfuros, y se explica su mecanismo como la competencia en la formación de óxidos formadores de capas protectoras en sitios localizados y la formación de capas salinas de los iones agresivos (3).

\section{DESARROLLO EXPERIMENTAL}

\subsection{Curvas de polarización anódica con inversión del barrido de potencial}

Las curvas de polarización anódica con inversión del barrido de potencial (4) permiten determinar valores críticos de potencial, como son el potencial de picado $\left(E_{\mathrm{p}}\right)$ y el potencial de repasivación de las picaduras $\left(E_{\mathrm{r}}\right)$.

Experimentalmente, las curvas de polarización anódica con inversión del barrido de potencial se obtienen realizando un barrido potenciodinámico o potenciostático a partir del potencial de corrosión, o de un potencial un poco inferior a éste, en dirección anódica, hasta detectar los hechos que se desean analizar; por ejemplo, la pasivación y la transpasivación del electrodo, invirtiendo luego el sentido del barrido de potencial, para observar si se produce o no histéresis y la importancia de la misma, u otras alteraciones de la curva relacionadas con el proceso de corrosión.

Cuando se estudian metales pasivables y se produce la destrucción local de la película pasivante, se inicia la corrosión por picaduras y crece rápidamente la densidad de corrriente antes de llegar a la zona transpasiva, como se esquematiza en la figura 2.

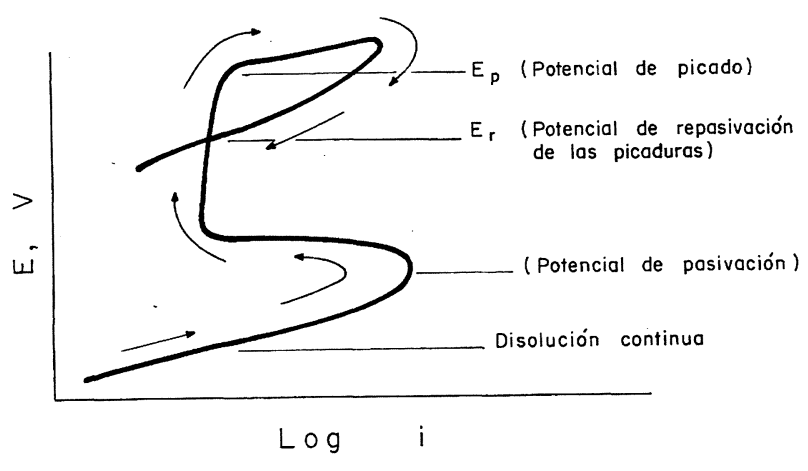

FIG. 2.- Curva de polarización con inversión del barrido de potencial (4).

Fig. 2.-Reverse sweep polarisation curve (4).

Con materiales susceptibles a la corrosión por picaduras, al invertir el barrido de potencial, la curva presenta una histéresis muy acentuada, efectuando el retorno a una densidad de corriente muy superior a la del primer tramo. El potencial al que se inicia el crecimiento rápido de la densidad de corriente se denomina potencial de picado $\left(E_{\mathrm{p}}\right)$ y el potencial en el que la curva de retorno cruza la rama pasiva se conoce como potencial de repasivación $\left(E_{\mathrm{r}}\right)$. Estos valores sirven como criterio electroquímico para caracterizar la resistencia de un determinado metal a la corrosión por picaduras (5).

Si se alcanza la zona transpasiva sin iniciarse la corrosión por picaduras se admite que el material no es susceptible a este tipo de ataque.

\subsection{Electrodos, equipo y soluciones}

Para poder realizar las pruebas electroquímicas se construyeron electrodos que consisten en encapsular en resina poliéster una muestra de cobre comercial del 99,94\% de pureza, exponiendo a la acción del medio una superficie de $1 \mathrm{~cm}^{2}$ del metal; dicha superficie expuesta fue lijada hasta lija 1200 y desengrasada con acetona, contando dicho electrodo con una conexión eléctrica aislada para la interfase electroquímica (Schlumberger 1286). Este es un equipo que controla el potencial de la muestra espécimen con respecto a un electrodo de referencia. Dicha interfase electroquímica se comunica a un ordenador personal por medio del puerto serie (RS232). Como electrodo de referencia se empleó el de calomelanos saturado (ECS) y como contraelectrodo una barra de grafito. La variación de potencial fue de $20 \mathrm{mV}$ cada $6 \mathrm{~s}$. Todas las experiencias se realizaron a temperatura ambiente.

Para llevar a cabo las diferentes pruebas y utilizando reactivos analíticos se prepararon las siguientes soluciones: 
Agua de mar según la norma británica B.S. 2900

\begin{tabular}{|c|c|}
\hline $\mathrm{NaCl}$ & 26 \\
\hline $\mathrm{MgCl}_{2}$ & 2,4 \\
\hline $\mathrm{MgSO}_{4}$ & 3,3 \\
\hline $\mathrm{KCl}$ & 0,73 \\
\hline $\mathrm{NaHCO}_{3}$ & 0,2 \\
\hline $\mathrm{NaBr}$ & 0,28 \\
\hline $\mathrm{CaCl}_{2}$ & 1,1 \\
\hline $\mathrm{pH}$ & 6,4 \\
\hline
\end{tabular}

Solución de $\mathrm{NaCl}$ y $\mathrm{Na}_{2} \mathrm{SO}_{4}$ con una concentración de $\mathrm{Cl}^{-}$y $\mathrm{SO}_{4}^{2-}$ similar a la del agua de mar sintética.

$\begin{array}{lcc}\mathrm{NaCl} & 26,5 & \mathrm{~g} / \mathrm{l} \\ \mathrm{Na}_{2} \mathrm{SO}_{4} & 3,89 & \mathrm{~g} / \mathrm{l} \\ \mathrm{pH} & 6,3 & \end{array}$

Para apreciar el efecto de la concentración de las especies $\mathrm{Cl}^{-}$y $\mathrm{SO}_{4}^{2-}$ sobre el fenómeno de la corrosión del cobre, se prepararon las soluciones que se presentan en la tabla I.

\section{RESULTADOS EXPERIMENTALES Y DISCUSIÓN}

Dado que el principal componente agresivo del agua de mar es el $\mathrm{NaCl}$, se realizaron una serie de curvas de polarización con inversión del barrido de potencial, variando en la solución la concentración de este componente (Figs. 3 a 6).

TABLA I.- Soluciones $\mathrm{NaCl} / \mathrm{Na}_{2} \mathrm{SO}_{4}$

TABLE I. $-\mathrm{NaCl} / \mathrm{Na}_{2} \mathrm{SO}_{4}$

\begin{tabular}{|c|c|c|c|l|}
\hline Solución & $\begin{array}{c}\text { Relación } \\
\mathrm{g} \mathrm{NaCl}^{2} \\
\mathrm{~g} \mathrm{Na}_{2} \mathrm{SO}_{4}\end{array}$ & $\begin{array}{c}\mathrm{NaCl}, \\
\mathrm{g} / \mathrm{l}\end{array}$ & $\begin{array}{c}\mathrm{Na}_{2} \mathrm{SO}_{4}, \\
\mathrm{~g} / \mathrm{l}\end{array}$ & $\mathrm{pH}$ \\
\hline 1 & 1,7 & 6,62 & 3,89 & 6,5 \\
2 & 2,17 & 8,48 & 3,89 & 6,8 \\
3 & 2,72 & 10,6 & 3,89 & 6,5 \\
4 & 3,4 & 13,25 & 3,89 & 6,5 \\
5 & 5,1 & 19,87 & 3,89 & 6,8 \\
6 & 6,8 & 26,5 & 3,89 & 6,5 \\
7 & 15 & 26,5 & 1,75 & 6,4 \\
8 & 20 & 26,5 & 1,32 & 7 \\
9 & 25 & 26,5 & 1,05 & 6,7 \\
10 & 30 & 26,5 & 0,855 & 6,8 \\
11 & 37,8 & 26,5 & 0,70 & 6,5 \\
12 & 40,1 & 26,5 & 0,661 & 6,5 \\
13 & 41,3 & 26,5 & 0,641 & 6,5 \\
\hline
\end{tabular}

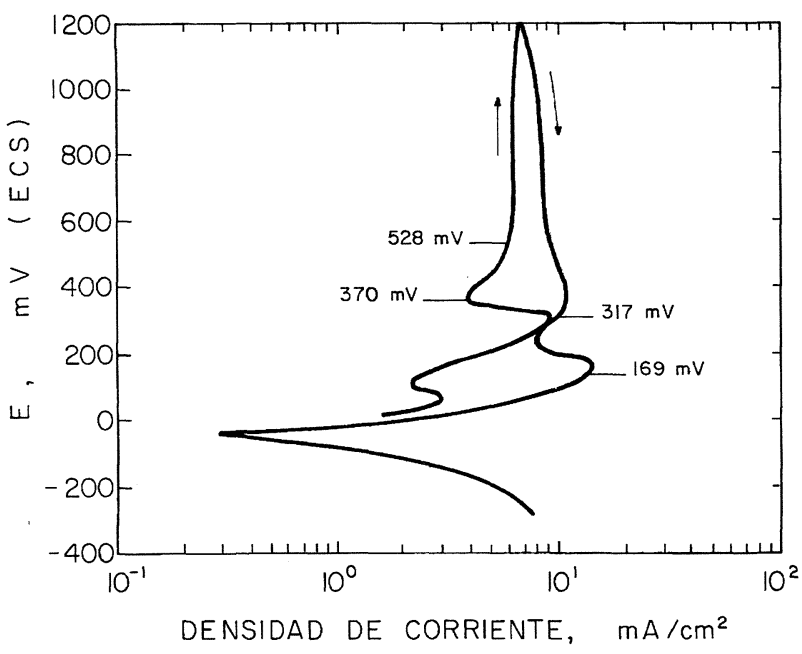

FIG. 3.- Curva de polarización del cobre en $\mathrm{NaCl}$ al $3 \%$. $\mathrm{pH}=6,4$.

Fig. 3.-Polarisation curve of copper in $3 \% \mathrm{NaCl}$ solution. $p H=6,4$.

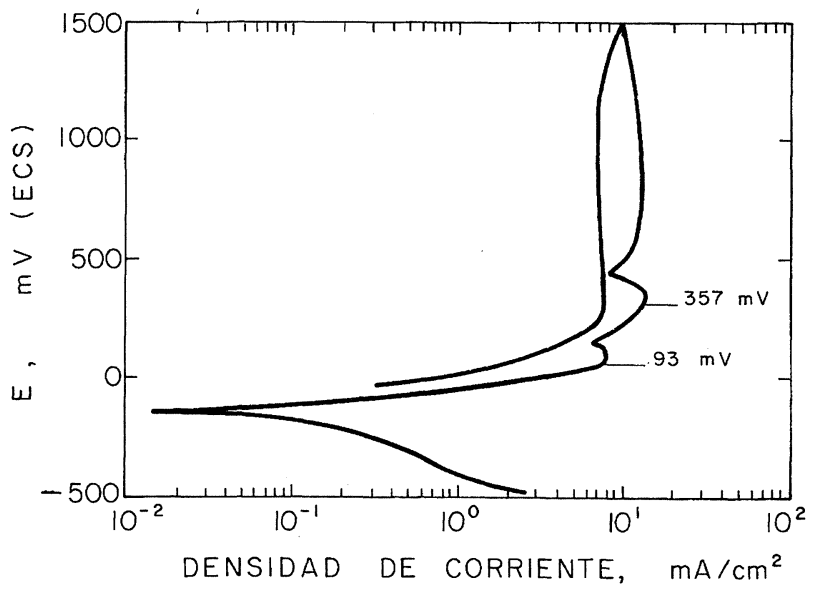

FIG. 4.- Curva de polarización del cobre en $\mathrm{NaCl}$ al $3 \%(13,25 \mathrm{~g} / \mathrm{l}) \cdot \mathrm{pH}=6,4$.

Fig. 4.-Polarisation curve of copper in $3 \% \mathrm{NaCl}$ $(13.25 \mathrm{~g} / \mathrm{l}) . \mathrm{pH}=6.4$.

Se aprecia que existen diferencias importantes al cambiar la concentración de $\mathrm{NaCl}$. Con concentraciones mayores del $3 \%$ (Fig. 3), se presentan dos picos de corriente y un potencial crítico de picado a $370 \mathrm{mV}$; en el regreso de potencial se observa un fenómeno de histéresis con valores de corriente superiores. Estos dos picos de corriente corresponden, el primero, a $160 \mathrm{mV}$, a la formación de $\mathrm{Cu}_{2} \mathrm{O}$ y el segundo, a $317 \mathrm{mV}$, a $\mathrm{Cu}_{2}\left(\mathrm{OH}_{3}\right) \mathrm{Cl}$, como ya indicaron Kato et al. (6) para cobre en soluciones de $\mathrm{NaCl}$ a $\mathrm{pHs}$ ligeramente ácidos o en soluciones neutras.

Al disminuir la concentración de $\mathrm{NaCl}$ (Figs. 4 a 6), se observa claramente que, con valores de corriente mayores, el fenómeno de histéresis 


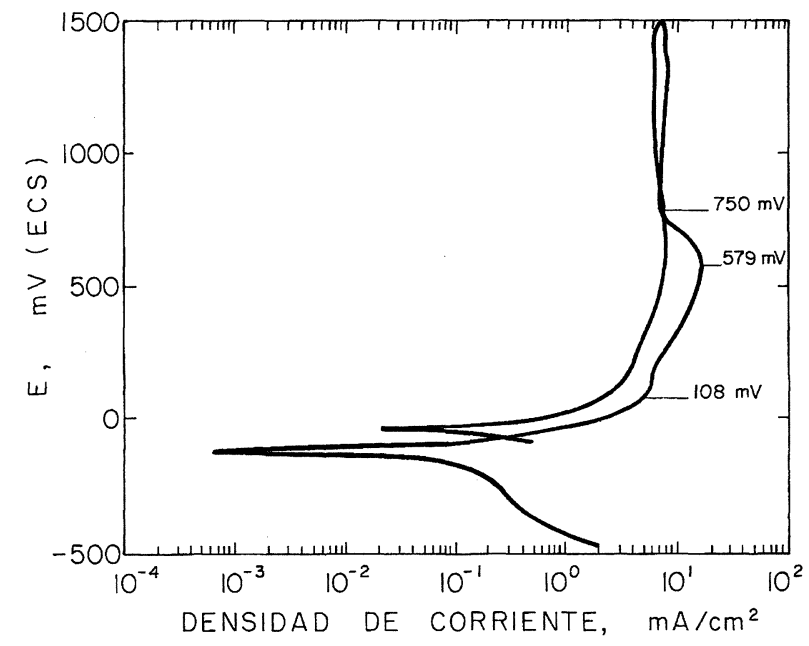

FIG. 5.- Curva de polarización del cobre en $\mathrm{NaCl}$ $(6,62 \mathrm{~g} / \mathrm{l}) \cdot \mathrm{pH}=6,4$.

Fig. 5.-Polarisation curve of copper in $\mathrm{NaCl}$ $(6.62 \mathrm{~g} / \mathrm{l}) . \mathrm{pH}=6.4$.

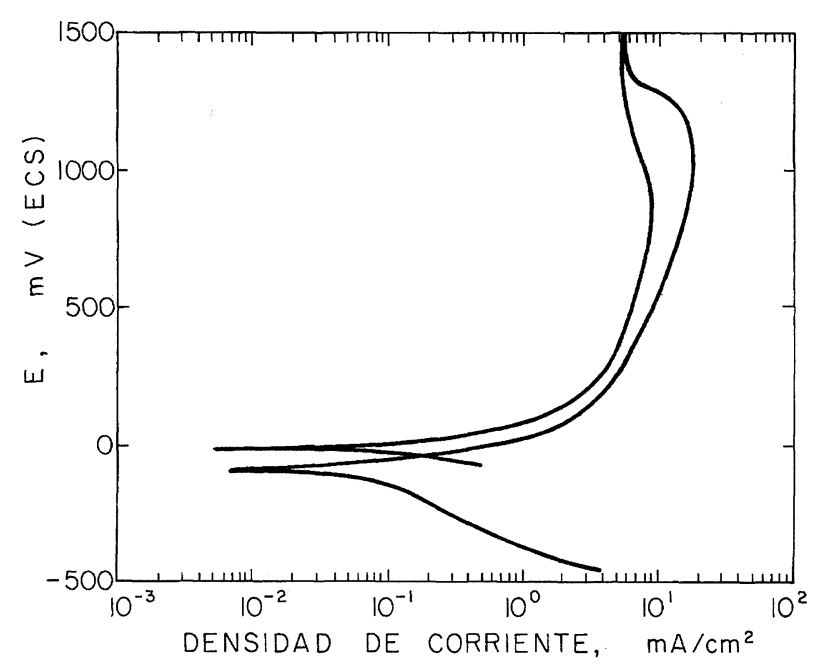

FIG. 6.- Curva de polarización del cobre en $\mathrm{NaCl}$ $(3,31 \mathrm{~g} / \mathrm{l}) \cdot \mathrm{pH}=6,4$.

Fig. 6.- Polarisation curve of copper in $\mathrm{NaCl}$ $(3.31 \mathrm{~g} / \mathrm{l}) . \mathrm{pH}=6.4$.

desaparece, indicando claramente que la capa de productos de corrosión es mucho más estable y no sufre una destrucción que se refleje en un incremento del área de exposición. Otro efecto muy significativo al disminuir la concentración de $\mathrm{NaCl}$ es la tendencia a desaparecer los picos de corriente, que se observan claramente cuando la concentración de cloruros es máxima. Esto permite concluir que la concentración de cloruros tiene relación directa con la formación de los productos de corrosión que se depositan en la superficie y con el nivel de protección que presentan.
En la figura 7, que corresponde a una curva de polarización de inversión del barrido de potencial de cobre en agua de mar sintética, se aprecia claramente un potencial crítico de picado de $502 \mathrm{mV}$ y un fenómeno de histéresis con valores de corriente superiores a los iniciales. Todo ello indica que el fenómeno de corrosión por picaduras se presenta sobre el cobre en este medio.

Lo que se pone de manifiesto es que algunos de los componentes del agua de mar tienen un efecto muy importante sobre el fenómeno de la corrosión por picaduras en el cobre.

Para poder determinar los agentes causantes del fenómeno de corrosión por picado en el cobre, se realizaron una serie de curvas de polarización con inversión del barrido de potencial en agua de mar, eliminando algunos de sus componentes.

Queda de manifiesto que en las curvas de polarización con inversión del barrido de potencial en que se encontraba ausente el $\mathrm{MgSO}_{4}$, por ejemplo, las recogidas en la figura 8 , no se presenta potencial crítico de picado, ni fenómeno de histéresis. Esto sugiere que el $\mathrm{MgSO}_{4}$ influye en el fenómeno de corrosión por picaduras del cobre.

Para determinar el grado de importancia de esta sal, se realizó una curva de polarización con inversión del barrido de potencial de cobre en una solución de $\mathrm{MgSO}_{4}$ con una concentración similar a la del agua de mar sintética (Fig. 9). Se observó que, por sí sólo, este compuesto no es capaz de provocar picaduras en el cobre, por lo que puede suponerse que es una combinación de sales la que ocasiona este fenómeno.

Para determinar lo anterior, se procedió a realizar una curva de polarización con inversión del

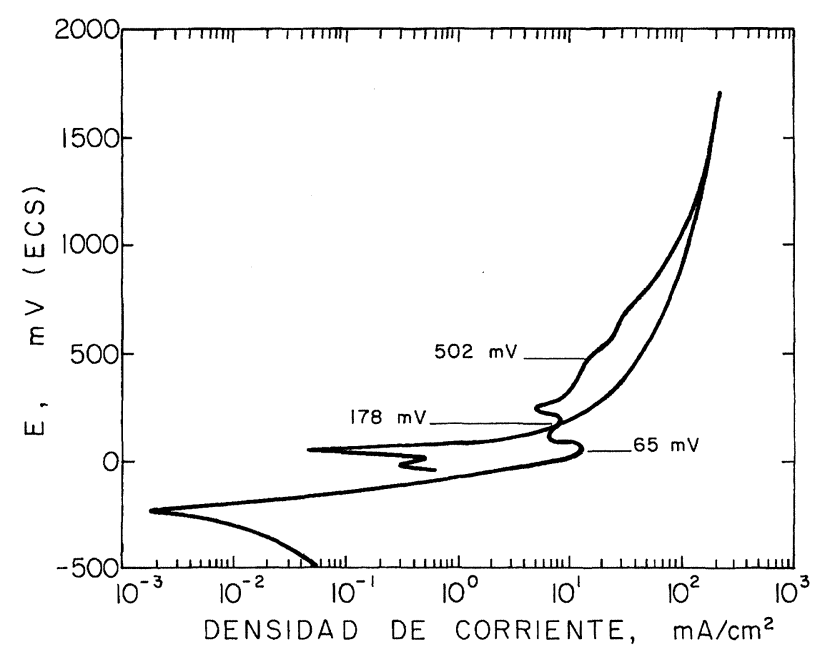

Fig. 7.- Curva de polarización del cobre en agua de mar sintética. $\mathrm{pH}=6,5$.

Fig. 7.- Polarisation curve of copper in artificial sea water. $p H=6.5$. 


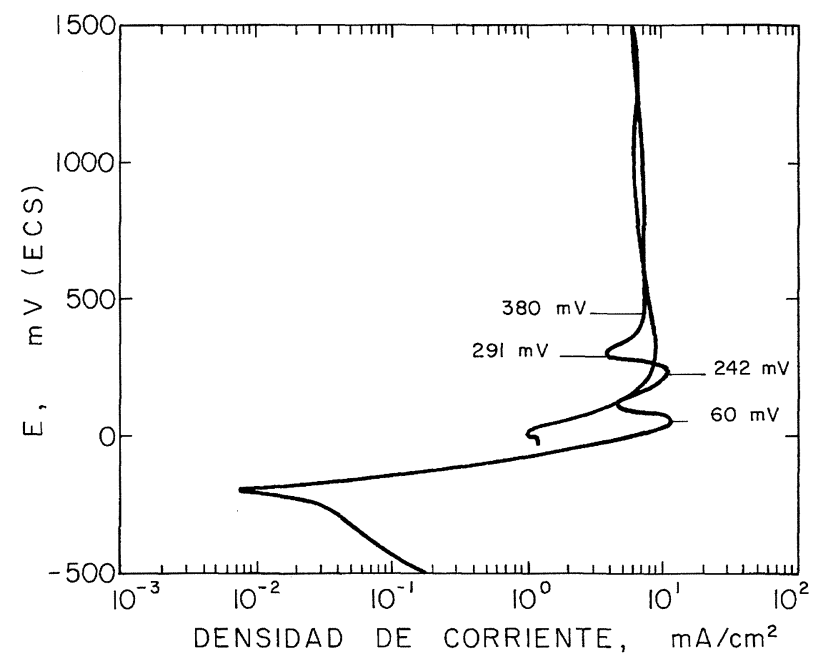

FIG. 8.- Curva de polarización del cobre en agua de mar sintética sin $\mathrm{MgSO}_{4}$ ni $\mathrm{MgCl}_{2} \cdot \mathrm{pH}=6,5$.

Fig. 8.- Polarisation curve of copper in artificial sea water without $\mathrm{MgSO}_{4}$ and $\mathrm{MgCl}_{2} . \mathrm{pH}=6.5$.

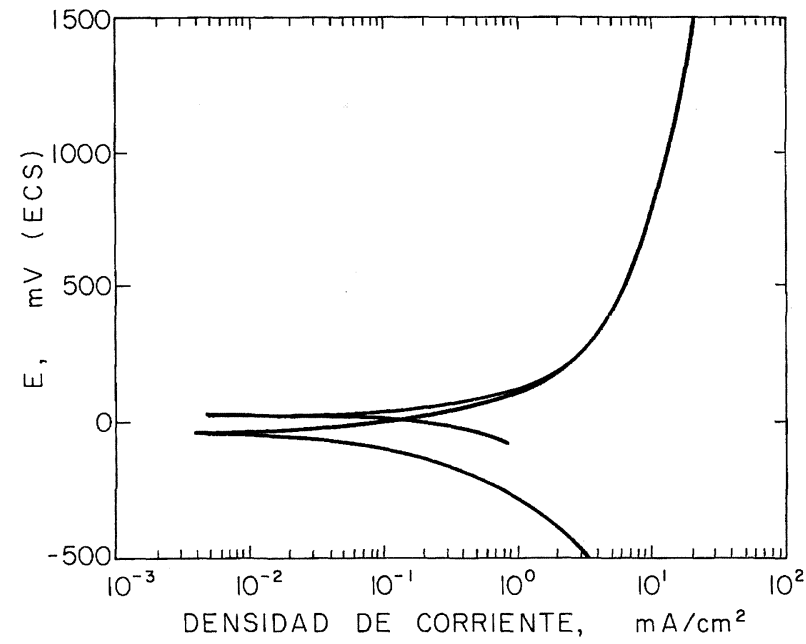

FIG. 9.- Curva de polarización del cobre en $\mathrm{MgSO}_{4}(3,3 \mathrm{~g} / \mathrm{l}) \cdot \mathrm{pH}=6,5$.

Fig. 9.- Polarisation curve of copper in $\mathrm{MgSO}_{4}$ solution $(3.3 \mathrm{~g} / \mathrm{l}) . \mathrm{pH}=6.5$.

barrido de potencial de cobre en una solución que contenía $\mathrm{NaCl}$ y $\mathrm{MgSO}_{4}$ en una concentración similar a la del agua de mar sintética (Fig 10). Se observa claramente un potencial crítico de picado a 555 $\mathrm{mV}$ y un fenómeno de histéresis, lo que indica la susceptibilidad del cobre a las picaduras en este medio.

Se pone de manifiesto que el fenómeno de corrosión por picaduras del cobre en agua de mar parece ser un fenómeno sinérgico entre el $\mathrm{NaCl}$ y el $\mathrm{MgSO}_{4}$, dado que estas sales, por sí mismas, no son capaces de ocasionar un fenómeno tan enérgico de picaduras en el cobre.

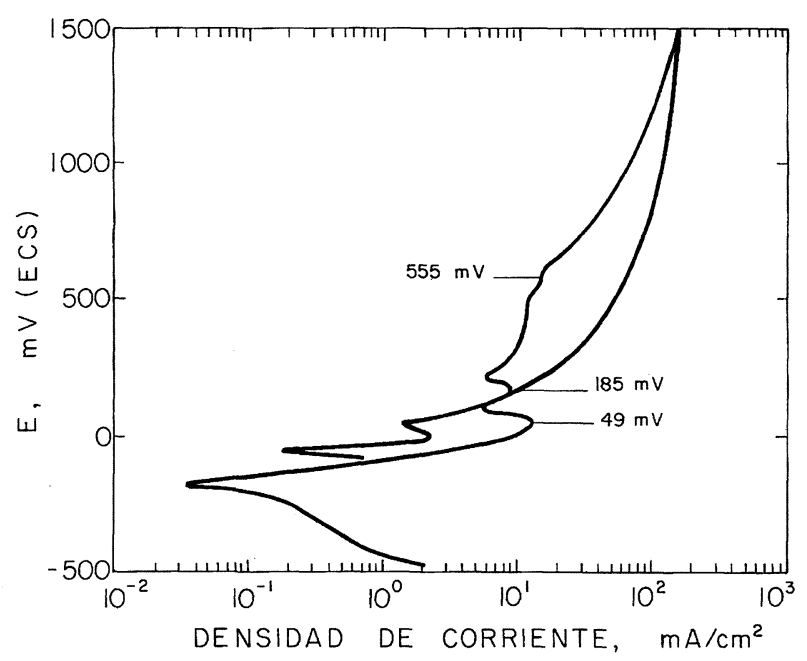

Fig. 10.- Curva de polarización del cobre en $\mathrm{NaCl}$ $(26,5 \mathrm{~g} / \mathrm{l})$ y $\mathrm{MgSO}_{4}(3,3 \mathrm{~g} / \mathrm{l}) . \mathrm{pH}=6,5$.

Fig. 10.- Polarisation curve of copper in $\mathrm{NaCl}$ $(26.5 \mathrm{~g} / \mathrm{l})$ and $\mathrm{MgSO}_{4}(3.3 \mathrm{~g} / \mathrm{l})$ solution. $\mathrm{pH}=6.5$.

En la figura 11 se presenta una curva de polarización con inversión del barrido de potencial de cobre en una solución de $\mathrm{NaCl}$ y $\mathrm{Na}_{2} \mathrm{SO}_{4}$, cuya concentración de iones $\mathrm{Cl}^{-}$y $\mathrm{SO}_{4}^{2-}$ es similar a la del agua de mar sintética. Se aprecia claramente un potencial crítico de picado de $582 \mathrm{mV}$ y un fenómeno de histéresis, indicando que la participación de los aniones de estas sales es determinante para el fenómeno de corrosión por picaduras en el cobre.

En vista de la importancia de los iones $\mathrm{Cl}^{-}$y los $\mathrm{SO}_{4}^{2-}$ en el fenómeno de corrosión por picaduras en el cobre, se realizó una serie de pruebas de barrido de potencial variando la concentración relativa de

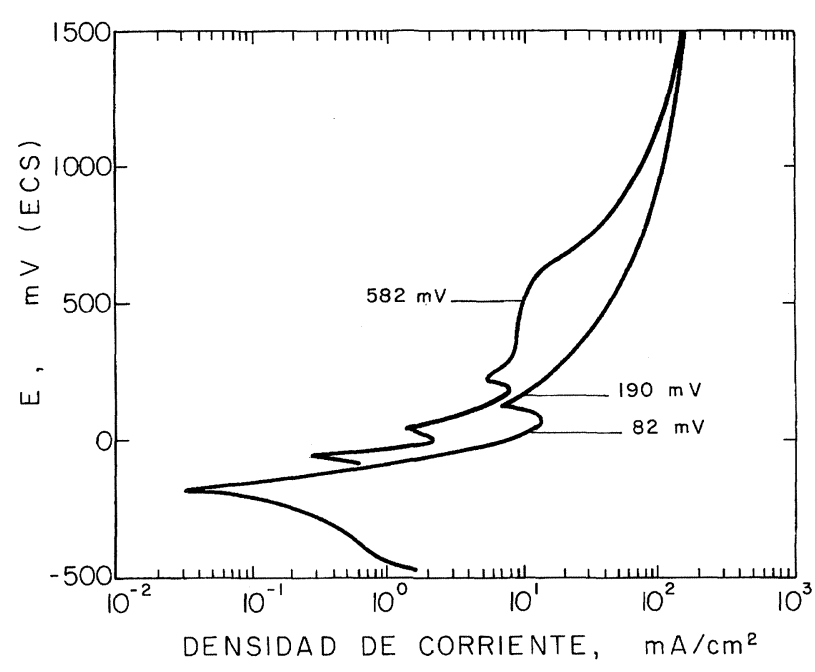

FIG. 11.- Curva de polarización del cobre en $\mathrm{NaCl}$ $(26,5 \mathrm{~g} / \mathrm{l})$ y $\mathrm{Na}_{2} \mathrm{SO}_{4}(3,89 \mathrm{~g} / \mathrm{l}) . \mathrm{pH}=6,5$.

Fig. 11.-Polarisation curve of copper in $\mathrm{NaCl}$ $(26.5 \mathrm{~g} / \mathrm{l})$ and $\mathrm{Na}_{2} \mathrm{SO}_{4}(3.89 \mathrm{~g} / \mathrm{l})$ solution, $\mathrm{pH} 6.5$ 
estos aniones para observar el cambio de potencial crítico de picado. Como se ve en la figura 12, existe una relación entre las concentraciones relativas de estos aniones y el potencial de picado, la cual se puede expresar por la siguiente ecuación de una parábola que se ajustó a los datos experimentales y que tiene un factor de correlación del $92 \%$.

$$
\begin{aligned}
E_{\mathrm{p}}= & 0,79\left(\mathrm{NaCl} / \mathrm{Na}_{2} \mathrm{SO}_{4}\right)^{2}-35,9773 \\
& \left(\mathrm{NaCl} / \mathrm{Na}_{2} \mathrm{SO}_{4}\right)+795,81 \ldots
\end{aligned}
$$

donde $E_{\mathrm{p}}$ es el potencial crítico de picado y $\left(\mathrm{NaCl} / \mathrm{Na}_{2} \mathrm{SO}_{4}\right)$ la relación en gramos entre estas dos sales. El comportamiento parabólico que se observa de esta ecuación empírica es típico de un fenómeno sinergético.

Retomando las curvas de polarización en agua de mar (Fig. 7) y la de una solución de $\mathrm{NaCl}$ y $\mathrm{MgSO}_{4}$ similar en concentración al agua de mar sintética (Fig. 10), se aprecia, en ambas curvas de polarización la presencia de dos picos de corriente a potenciales similares, lo cual indica que básicamente se trata de los mismos compuestos formados.

El primer pico de corriente correspondería a la formación de $\mathrm{Cu}_{2} \mathrm{O}$ como indicaron Kato et al. (6), el segundo correspondería a la formación de sulfato básico de cobre, denominado Brochantita, cuya fórmula es $\mathrm{CuSO} 4 \cdot 3 \mathrm{Cu}(\mathrm{OH})_{2} \cdot \mathrm{H}_{2} \mathrm{O}$. Estos resultados fueron confirmados mediante análisis de difracción de rayos X por Shalaby (7).

La figura 13 corresponde a la superficie picada del cobre en agua de mar sintética con los produc-

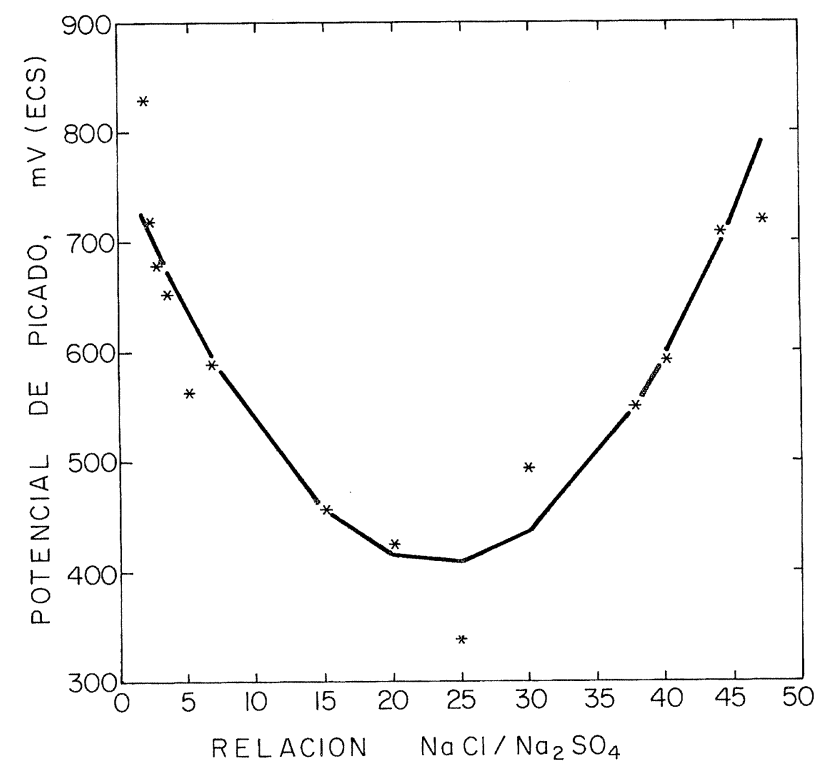

FIG. 12.- Variación del potencial de picado con la relación $\mathrm{NaCl} / \mathrm{Na}_{2} \mathrm{SO}_{4}$.

Fig. 12.- Relation between the pitting potential of copper and the $\mathrm{NaCl} / \mathrm{Na}_{2} \mathrm{SO}_{4}$ by weight ratio.

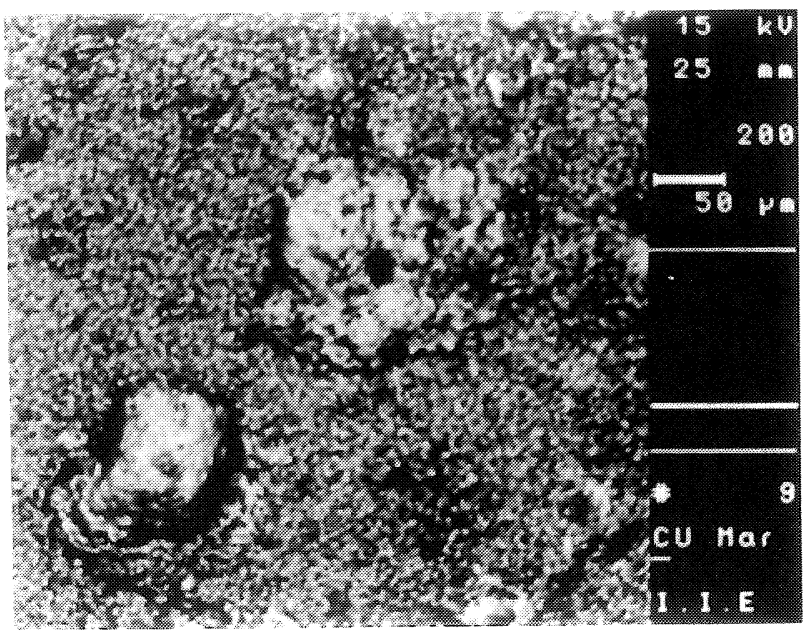

FIG. 13.- Superficie picada de cobre en agua de $\operatorname{mar}(\times 200)$.

Fig. 13.- Pitted surface of copper in sea water $(\times 200)$.

tos de corrosión. Se puede ver la presencia de promontorios que, en algunos casos, presentan un cráter, lo cual corresponde a una picadura, observándose que toda picadura tiene asociado uno de estos promontorios, pero no todos los promontorios tienen asociada una picadura. Esta misma morfología de picado en el cobre fue apreciada por Shalaby (7).

La figura 14 corresponde a un microanálisis de azufre, donde se aprecia claramente que el sulfato básico de cobre, la brochantita, se deposita en toda la superficie del metal, con excepción de aquellos lugares donde está presente un promontorio.

En cambio, en la figura 15, que corresponde a un microanálisis de cloro, se aprecia claramente

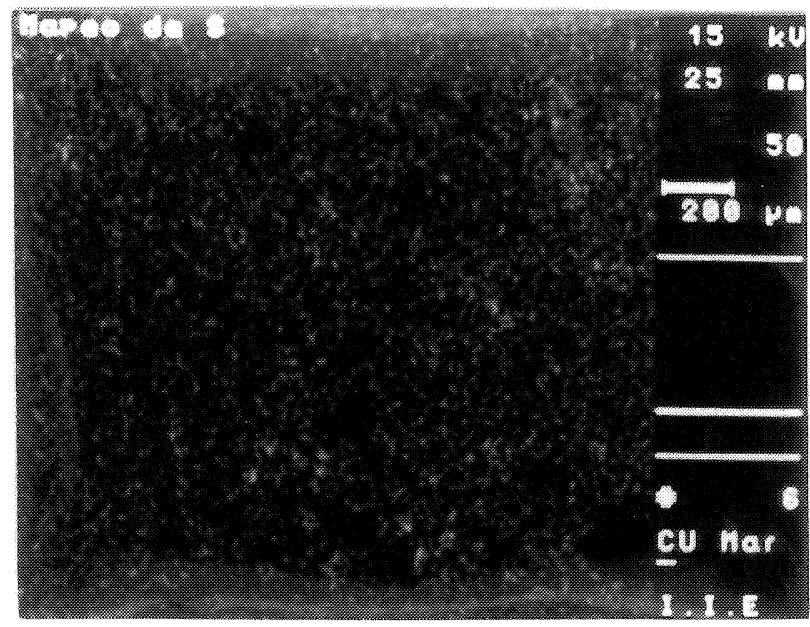

FIG. 14.- Distribución de azufre en la superficie picada del cobre en agua de mar $(\times 50)$.

Fig. 14.- Mapping of sulphur over the pitted surface of copper in sea water $(\times 50)$. 


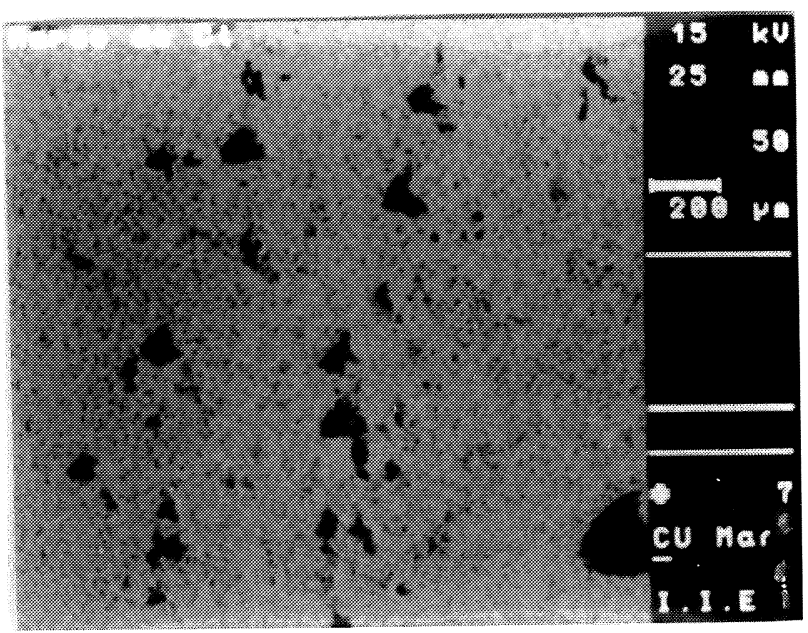

FIG. 15.- Distribución de cloro en la superficie picada del cobre en agua de mar $(\times 50)$.

Fig. 15.- Mapping of chlorine over the pitted surface of copper in sea water $(\times 50)$.

una distribución uniforme en la superficie, mientras que los promontorios se ven enriquecidos por compuestos clorados, indicando esto los sitios idóneos para la formación de las picaduras.

\section{CONCLUSIONES}

De esta investigación se puede concluir que las soluciones de $\mathrm{NaCl}$ al $3 \%$ no son un buen sustituti- vo del agua de mar. En cambio, empleando agua de mar sintética se pudo determinar que el cobre es susceptible a corrosión por picaduras en agua de mar, por la interacción, principalmente, de los iones cloruro y sulfato, indicando claramente que es un fenómeno de sinergia entre estos dos iones.

Se ha encontrado, asimismo, una relación entre el potencial crítico de picado $\left(E_{\mathrm{p}}\right)$ y la relación $\mathrm{Cl}^{-} / \mathrm{SO}_{4}^{2-}$, que se ajusta a un comportamiento parabólico, de acuerdo con la ecuación (5).

\section{REFERENCIAS}

(1) CAmbell, H.S. A Review: Pitting Corrosion of Copper and its Alloys, Localized Corrosion NACE-3. R. STAEHLE, B. Brown, J. Kruger, A. Agrawal, Eds., National Association of Corrosion Engineering, Houston, 1974: 625.

(2) Cornwell, F.J., Wildsmith, G., Gilbert.P.T. Brit. Corros. $J .$, 8, 1973: 202.

(3) Figueroa, M.G., Salvarezza, R.C. y Arvia, A.J. Electrochim. Acta, 31 (6) 1986: 665.

(4) Pourbaix M. Proceedings of the Conference on Localized Corrosion. NACE, Houston, 1974.

(5) Smialowska, S. y Czachor, W. Corros. Sci., 11 (12), 1971: 901.

(6) Kato, C. y Pickering, H.W. J. Electrochem. Soc., 131, 1984: 1219.

(7) Shalaby, H.M., Al-Kharafi, F.M. y Gouda, V.K. Corrosion (NACE), 45 (7), 1989: 536. 